\title{
Structure and kinematics of the Virgo cluster of galaxies *
}

\author{
Olga G. Kashibadze ${ }^{1, \star \star}$, Igor D. Karachentsev ${ }^{1}$, and Valentina E. Karachentseva ${ }^{2}$ \\ ${ }^{1}$ Special Astrophysical Observatory of the Russian Academy of Sciences, Nizhnij Arkhyz, Karachay-Cherkessia 369167, Russia \\ e-mail: phiruzi@gmail.com \\ 2 Main Astronomical Observatory of the National Academy of Sciences, 27 Akademika Zabolotnoho St., Kyiv 03143, Ukraine \\ Received 25 June 2019 / Accepted 24 January 2020
}

\begin{abstract}
Aims. This work considers the Virgo cluster of galaxies, focusing on its structure, kinematics, and morphological landscape. Our principal aim is to estimate the virial mass of the cluster. For this purpose, we present a sample of 1537 galaxies with radial velocities $V_{\mathrm{LG}}<2600 \mathrm{~km} \mathrm{~s}^{-1}$ situated within a region of $\Delta \mathrm{SGL}=30^{\circ}$ and $\Delta \mathrm{SGB}=20^{\circ}$ around $\mathrm{M} 87$. About half of the galaxies have distance estimates.

Methods. We selected 398 galaxies with distances in a (17 \pm 5$)$ Mpc range. Based on their 1D and 2D number-density profiles and their radial velocity dispersions, we made an estimate for the virial mass of the Virgo cluster.

Results. We identify the infall of galaxies towards the Virgo cluster core along the Virgo Southern Extension filament. From a 1D profile of the cluster, we obtain the virial mass estimate of $(6.3 \pm 0.9) \times 10^{14} M_{\odot}$, which is in tight agreement with its mass estimate via the external infall pattern of galaxies.

Conclusions. We conclude that the Virgo cluster outskirts between the virial radius and the zero-velocity radius do not contain significant amounts of dark matter beyond the virial radius.
\end{abstract}

Key words. galaxies: clusters: individual: Virgo

\section{Introduction}

It is well known that a thousand of the brightest galaxies with apparent magnitudes $m_{B}<13^{\mathrm{m}}$ (Sandage \& Tammann 1981) form a planar structure across the sky, called the Local Supercluster (de Vaucouleurs 1961). The most dense region of the Local Supercluster (LSC) is the Virgo cluster of galaxies, with the centre usually identified by the radiogalaxy Virgo $\mathrm{A}=\mathrm{M} 87=$ NGC 4486.

In our previous paper (Kashibadze et al. 2018, KKK18), we considered the distribution and the radial velocity field of galaxies located within the equatorial belt of $100^{\circ}$ by $20^{\circ}$, centred on M 87 with its supergalactic coordinates $\mathrm{SGL}=102.88^{\circ}$ and $\mathrm{SGB}=-2.35^{\circ}$. This region, including the whole Virgo cluster and its spurs, contains 2158 galaxies with radial velocities $V_{\mathrm{LG}}<$ $2000 \mathrm{~km} \mathrm{~s}^{-1}$ relative to the Local group centroid. Half of them have distance estimates $D$ and peculiar velocities $V_{\text {pec }}=V_{\mathrm{LG}}-$ $H_{0} \times D$, where $H_{0}$ is the local Hubble parameter, which is set to $73 \mathrm{~km} \mathrm{~s}^{-1} \mathrm{Mpc}^{-1}$. It has been found out that some galaxy groups in the belt have peculiar velocities up to $\sim(500-800) \mathrm{km} \mathrm{s}^{-1}$, comparable with virial velocities in rich clusters of galaxies. Using the spherically symmetrical infall model, we estimated the radius of the zero-velocity surface of the Virgo cluster as $23^{\circ}$, or $(7.8 \pm 0.3) \mathrm{Mpc}$, corresponding to the total mass of the cluster $M_{\mathrm{T}}=(7.4 \pm 0.9) \times 10^{14} M_{\odot}$. Shaya et al. (2017) obtained nearly the same value of the radius of the zero-velocity surface, $(7.3 \pm 0.3) \mathrm{Mpc}$, considering trajectories of galaxies within the Numerical Action Method.

\footnotetext{
* Full Table 1 is only available at the CDS via anonymous ftp to cdsarc.u-strasbg. fr (130.79.128.5) or via http://cdsarc. u-strasbg. fr/viz-bin/cat/J/A+A/635/A135

$\star \star$ f.k.a. Nasonova.
}

In this work, which is a continuation of the previous KKK18 study, we restrict our analysis with the region limited by $\triangle \mathrm{SGL}=$ $30^{\circ}$ and $\triangle \mathrm{SGB}=20^{\circ}$ centred at M 87 . The sample used in KKK18 was supplemented by galaxies with radial velocities $V_{\mathrm{LG}}=[2000-2600] \mathrm{km} \mathrm{s}^{-1}$ and new distances from Cantiello et al. (2018) and Toloba et al. (2018).

It should be noted that the Virgo Cluster Catalog (VCC, Binggeli et al. 1985) and the Extended Virgo Cluster Catalog (EVCC, Kim et al. 2014) have made a foundational contribution to studies of the structure of the Virgo cluster. Both catalogues have expanded our insights into the dwarf population of the nearest cluster of galaxies. Still, there are no measured radial velocities for most dwarf galaxies in the Virgo region, and their membership in the cluster is considered from some indirect indicators. Tonry et al. (2000) and Mei et al. (2007) performed bulk distance measurements for members of the Virgo cluster. The surface-brightness-fluctuation method they had used provided distances for more than a hundred of the most bright $\mathrm{E}$ and S0 galaxies with an accuracy of $\sim 10-15 \%$. The data are very important for understanding the structure and the dynamics of the Virgo cluster, since radial velocities of galaxies in the vicinity of a massive attractor are ambiguous and uncertain indicators of their distances.

The ALFALFA $H I$-survey of galaxies in Arecibo (Haynes et al. 2011, 2018) brought radial velocities and $H I$-line widths for many late-type galaxies in the Virgo cluster area. This allowed us to estimate new distances for 380 irregular and spiral galaxies in the sky region in question, doubling the total number of galaxies with known distances. This progress in distance estimates provided us with an opportunity to review the structure and dynamics of the Virgo cluster again. In total, we have a sample of 1537 galaxies inside the area of $30^{\circ}$ by $20^{\circ}$ with measured 


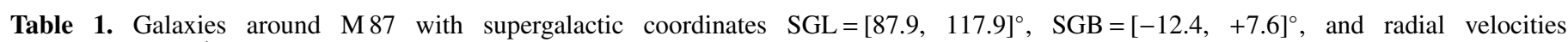
$V_{\mathrm{LG}}<2600 \mathrm{~km} \mathrm{~s}^{-1}$.

\begin{tabular}{|c|c|c|c|c|c|c|c|c|c|c|c|c|c|}
\hline Name & RA (2000) Dec & $\begin{array}{r}\text { SGL } \\
\left({ }^{\circ}\right)\end{array}$ & $\begin{array}{r}\text { SGB } \\
\left({ }^{\circ}\right)\end{array}$ & $\begin{array}{l}R_{\mathrm{p}} \\
\left({ }^{\circ}\right)\end{array}$ & $\begin{array}{r}V_{\mathrm{LG}} \\
\left(\mathrm{km} \mathrm{s}^{-1}\right)\end{array}$ & Type & $\begin{array}{c}B_{\mathrm{T}} \\
(\mathrm{mag})\end{array}$ & $\begin{array}{r}K \\
(\mathrm{mag})\end{array}$ & $\begin{array}{r}W_{50} \\
\left(\mathrm{~km} \mathrm{~s}^{-1}\right)\end{array}$ & $\begin{array}{r}m_{21} \\
(\mathrm{mag})\end{array}$ & $\begin{array}{r}m-M \\
(\mathrm{mag})\end{array}$ & $\begin{array}{r}D \\
(\mathrm{Mpc})\end{array}$ & Meth \\
\hline PGC1664006 & J113206.6+220731 & 88.93 & -12.33 & 17.05 & 1091 & Im & 17.36 & 15.01 & 47 & 18.08 & 31.28 & 18.00 & $\mathrm{TF}$ \\
\hline PGC1620526 & $\mathrm{J} 113554.4+201320$ & 91.08 & -12.24 & 15.31 & 1038 & I & 17.04 & 14.69 & 108 & 17.69 & $\ldots$ & $\ldots$ & \\
\hline GC1597887 & J113908.9+193460 & 91.97 & -11.77 & 14.34 & 1636 & I & 8.28 & 15.93 & $\ldots$ & & & $\ldots$ & \\
\hline UGC06670 & J114229.4+181959 & 93.45 & -11.49 & 13.08 & 823 & I & 3.56 & 11.21 & 194 & 14.60 & 31.29 & 18.10 & $\mathrm{TF}$ \\
\hline PGC1519757 & $\mathrm{J} 114440.7+165359$ & 95.01 & -11.51 & 12.04 & 813 & $\mathrm{BCD}$ & 16.98 & 14.63 & 91 & 17.97 & & $\ldots$ & \\
\hline UGC06747 & $\mathrm{J} 114624.0+134938$ & 98.11 & -12.18 & 10.91 & 2574 & $\mathrm{Sdm}$ & 16.27 & 13.67 & 96 & 15.30 & 31.53 & 20.30 & $\mathrm{TFb}$ \\
\hline EVCC2007 & $\mathrm{J} 114626.5+193012$ & 92.69 & -10.19 & 12.80 & 2086 & $\mathrm{Im}$ & & 16.81 & 102 & 17.66 & & 57.10 & $\mathrm{TFb}$ \\
\hline AGC219628 & $\mathrm{J} 114714.0+184028$ & 93.54 & -10.31 & 12.22 & 1746 & $\mathrm{Sm}$ & & & 77 & 17.49 & 32.73 & 35.10 & $\mathrm{TFb}$ \\
\hline SDSSJ114717 & $\mathrm{J} 114717.5+162005$ & 95.77 & -11 . & 11.2 & 700 & $\mathrm{E}$ & & & & & & $\ldots$ & \\
\hline PGC28 & $14816.4+183$ & 93.6 & -10 & 12. & & $\mathrm{Im}$ & & & 65 & 16.41 & & 18.24 & $\mathrm{TF}$ \\
\hline SDSs & $\mathrm{J} 1148$ & 95.08 & -10.50 & 11.2 & 963 & I & .38 & 15. & 42 & 17.72 & & 15.31 & $\mathrm{TF}$ \\
\hline $\mathrm{AGC}$ & $\mathrm{J} 114$ & 95.51 & -10.65 & 11.0 & 1510 & I & 3.60 & 16. & 72 & 17.18 & 32.64 & 33.85 & $\mathrm{TFb}$ \\
\hline & 16 & 88.79 & -8.09 & 15. & 452 & I & & 12. & 86 & 15.50 & 30.69 & 13.74 & bs \\
\hline 18400 & $\mathrm{~J} 11$ & 95.04 & -10.39 & 11. & 519 & $\mathrm{BCD}$ & 18.06 & 15. & $\ldots$ & & & & \\
\hline 541 & $\mathrm{~J} 114$ & 95.03 & -10.32 & 11.15 & 1266 & $\mathrm{Im}$ & 18.20 & 15. & 66 & 17.50 & 32.55 & 32.33 & $\mathrm{TFb}$ \\
\hline & $.0+151540$ & 96.99 & -10.98 & 10.42 & 747 & $\mathrm{E}$ & & 13. & $\ldots$ & & $\ldots$ & $\ldots$ & \\
\hline 14957 & $.1+161744$ & 96.03 & -10.53 & 10.64 & 1110 & I & 18.11 & 15.76 & 33 & 18.34 & 30.89 & 15.10 & $\mathrm{TF}$ \\
\hline PGC03 & $2.7+150124$ & 97.26 & -10.94 & 10.24 & 638 & $\mathrm{BCD}$ & 15.43 & 13.08 & 47 & 16.80 & 30.67 & 13.60 & $\mathrm{TF}$ \\
\hline PGC037048 & $\mathrm{J} 115055.9+143542$ & 97.74 & -10.88 & & 893 & $\operatorname{Im}$ & 15.52 & 13.17 & 52 & 15.70 & 30.32 & 11.60 & $\mathrm{TF}$ \\
\hline KIG0506 & $\mathrm{J} 115201.9+135244$ & 98.52 & -10.87 & 9.5 & 847 & I & 16.12 & 13.77 & 79 & 16.61 & 31.31 & 18.30 & $\mathrm{TF}$ \\
\hline SDSSJ115220 & $\mathrm{J} 115220.2+152736$ & 97.03 & -10.27 & 9.82 & 676 & $\mathrm{Im}$ & 17.53 & 15.18 & 68 & 17.65 & & & \\
\hline SDSSJ 115300 & $115300.3+160230$ & 96.53 & -9.93 & & & I & & & 23 & 19.93 & & & \\
\hline & 23 & 95. & -9. & & & I & & & 41 & 16.77 & & 11.59 & \\
\hline AG & $\mathrm{J} 115$ & 100. & -10 & & & I & & & 32 & 16. & & 8.20 & \\
\hline UGC & $\mathrm{J} 115$ & 92 & -8.1 & 11. & 522 & I & & & 90 & 16.20 & 31.07 & 16.40 & $\mathrm{TF}$ \\
\hline NGC & $4+064459$ & 105.74 & -12.22 & 10.2 & 2348 & $\mathrm{Sb}$ & 12.45 & 8.6 & 436 & 13.27 & 32.53 & 32.70 & $\mathrm{tf}$ \\
\hline PGC037490 & $\mathrm{J} 115602.5+064041$ & 105.81 & -12.23 & 10.30 & 2354 & So & 15.83 & 11.73 & $\ldots$ & & & $\ldots$ & \\
\hline AGC215716 & $\mathrm{J} 115707.2+064032$ & 105.90 & -11.97 & 10.08 & 2358 & $\mathrm{Sm}$ & 17.70 & 15.35 & 100 & 17.39 & 33.75 & 56.20 & TF \\
\hline PGC1488625 & J115840.4+153534 & 97.42 & -8.79 & 8.43 & 458 & I & 18.28 & 15.93 & $\ldots$ & $\ldots$ & $\ldots$ & $\ldots$ & \\
\hline AGC213178 & $\mathrm{J} 115900.7+044011$ & 108.00 & -12.12 & 11.01 & 1451 & $\mathrm{BCD}$ & 16.91 & 14.56 & 58 & 18.20 & 31.44 & 19.40 & $\mathrm{TF}$ \\
\hline PGC037779 & $\mathrm{J} 115933.8+135315$ & 99.12 & -9.14 & 7.75 & 1334 & $\mathrm{Sm}$ & 15.09 & 12.74 & 57 & 15.70 & 30.27 & 11.30 & $\mathrm{TF}$ \\
\hline PGC0 & $\mathrm{J} 115949.4+212656$ & 91.9 & -6.58 & 11.70 & 1893 & $\mathrm{Sm}$ & 15.81 & 13.46 & 124 & 16.02 & 32.77 & 35.81 & $\mathrm{TF}$ \\
\hline NGC4032 & $\mathrm{J} 120032.9+200426$ & 93.30 & -6 & 10.57 & 1186 & $\mathrm{Sb}$ & 13.03 & 9.18 & 107 & 14.28 & & & \\
\hline PGC1380373 & $\mathrm{J} 120056.1+102956$ & 102.49 & -9.90 & 7.5 & 2529 & $\mathrm{Im}$ & 16.44 & 14.09 & 83 & 16.84 & 32.12 & 26.50 & $\mathrm{TF}$ \\
\hline IC0755 & $\mathrm{J} 120110.5+140615$ & 99.04 & -8.70 & 7.41 & 1419 & $\mathrm{Sbc}$ & 14.09 & 10.49 & 206 & 14.73 & 32.34 & 29.30 & tf \\
\hline
\end{tabular}

Notes. The table contains 1537 galaxies with radial velocities $V_{\mathrm{LG}}<2600 \mathrm{~km} \mathrm{~s}^{-1}$. Among them, 738 galaxies have distance estimates: 358 from public databases and 380 distances estimated for the first time in this paper (indicated in the last column as TF or TFb). The full table is available at the CDS.

radial velocities. Among these galaxies, $738(48 \%)$ have distance estimates.

The paper is organised as follows: in Sect. 2, we present the sample selected for our analysis. The total luminosity of the Virgo cluster core in $B$ and $K$ bands is briefly summarised in Sect. 3. Distributions of early-type (E, S0, dSph), spiral (Sa-Sm) and irregular (I, Im, BCD) galaxies on the sky and in the depth are presented in Sect. 4. Section 5 describes 2D and 1D profiles of the Virgo cluster delineated by galaxies within a distance range of $(17 \pm 5) \mathrm{Mpc}$, and it presents various estimates of the virial mass of the cluster. The basic parameters of the Virgo cluster are fixed in Sect. 6. A brief summary of our results are given in Sect. 7. A Virgo cluster distance of 16.5 Mpc (Mei et al. 2007) is used throughout the paper, where $6^{\circ}=1.7 \mathrm{Mpc}$ is the cluster virial radius.

\section{The Virgo cluster core and suburbs}

The basic observational characteristics of 1537 galaxies in the considered area are presented in Table 1, with the full machine readable version available at SIMBAD Astronomical Database $^{1}$. The table columns contain the (1) galaxy name; (2) equatorial coordinates J2000.0; (3, 4) supergalactic coordinates; (5) projected distance of a galaxy from M 87; (6) radial velocity $\left(\mathrm{km} \mathrm{s}^{-1}\right)$ relative to the Local Group centroid; (7) morphological type determined by us based on images from Sloan Digital Sky Survey (SDSS, Abazajian et al. 2009) and Panoramic Survey Telescope and Rapid Response System (PanSTARRS, Chambers et al. 2016); (8) apparent $B$-magnitude from HyperLEDA ${ }^{2}$ (Makarov et al. 2014) or NASA Extragalactic Database ${ }^{3}$ (NED); (9) apparent $K$-magnitude calculated from $B$-magnitude adjusted for the morphological type of the galaxy according to Jarrett et al. (2003); (10) $21 \mathrm{~cm} \mathrm{HI} \mathrm{line} \mathrm{width}$ $\left(\mathrm{km} \mathrm{s}^{-1}\right)$ at half maximum from HyperLEDA data; (11) apparent HI-magnitude from HyperLEDA; $(12,13)$ distance modulus and galaxy distance (Mpc), (14) method applied to determine distance: "cep", "SN"-from supernovae and cepheids luminosity,

\footnotetext{
1 http://simbad.u-strasbg.fr/simbad/

2 http://leda.univ-lyon $1 . \mathrm{fr} /$

http://ned.ipac.caltech.edu/
} 


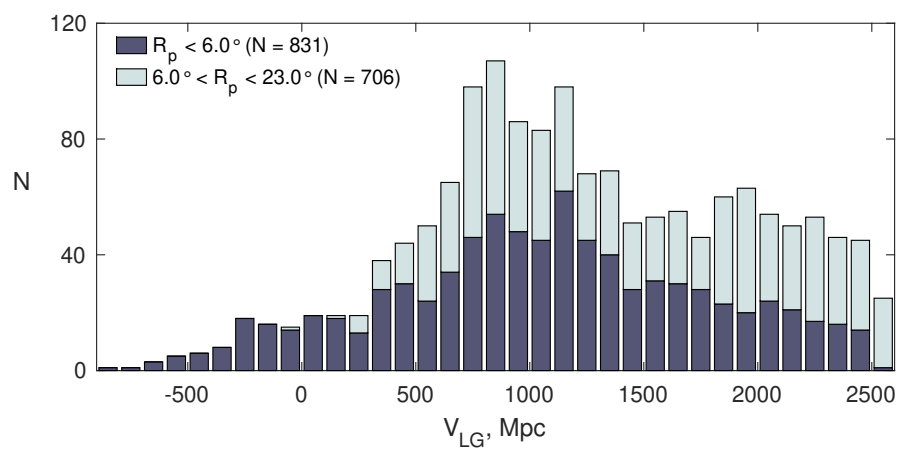

Fig. 1. Distribution of galaxies in Virgo cluster area by their radial velocities relative to the Local Group centroid. The virial core members are shown with a darker colour.

"sbf"-from surface brightness fluctuation, "rgb"-from the tip of the red giant branch, "bs"-from the brightest stars luminosity, "FP"-from the fundamental plane, "gc"-from luminosity function of globular clusters, "tf", "TF", "TFb"-from Tully \& Fisher (1977), with lowercase indicating distance estimates from NED, and uppercase indicating our estimates based on classical (Tully \& Pierce 2000) or baryonic (Karachentsev et al. 2017) TullyFisher relation.

The distribution of galaxies in the considered area by their radial velocities relative to the Local Group centroid is presented in Fig. 1. Galaxies populating the central virial zone of the cluster limited by $6.0^{\circ}$ are marked with a darker colour. Clearly, the members of the virial core of the cluster show quite a symmetrical distribution around the mean velocity of $\sim 1000 \mathrm{~km} \mathrm{~s}^{-1}$. The region of negative radial velocities is populated entirely by the virial core galaxies, providing evidence of nearly radial motions (Karachentsev \& Kashibadze 2010). The relative number of galaxies in the outskirts of the cluster grows with higher values of $V_{\mathrm{LG}}$ and becomes predominant with $V_{\mathrm{LG}}>1800 \mathrm{~km} \mathrm{~s}^{-1}$. Assuming the symmetry of the velocity distribution relative to the average, we estimate that $1-2 \%$ of the virial core members may lie beyond $V_{\mathrm{LG}}>2600 \mathrm{~km} \mathrm{~s}^{-1}$, being lost among field galaxies.

The upper panel of Fig. 2 reproduces the distribution of galaxies in our area by distances. The galaxies of the virial core are marked with a darker colour. About a half of the virial zone galaxies have distance estimates lying within (14-18) Mpc, suggesting their real membership in the Virgo cluster with its mean distance of $16.5 \pm 0.2 \mathrm{Mpc}$ according to Mei et al. (2007). Peripheral galaxies also show the peak at (14-20) Mpc, though far less distinctly. The significant amount of galaxies resides at distances $D>30 \mathrm{Mpc}$. Their presence in the $V_{\mathrm{LG}}<2600 \mathrm{~km} \mathrm{~s}^{-1} \mathrm{sam}-$ ple is partially caused by infall of background galaxies towards the centre of the attractor, as well as by the existence of a large expanding cosmic void between the Virgo and the Coma clusters, which imparts an additional velocity component towards an observer. Some galaxies in the larger $D$ tail area may be caught due to errors in distance estimates.

We combined our data into two groups: the first containing galaxies with accurately measured distances (sbf, cep, SN, $\mathrm{rgb}$ ), and the second including galaxies with less reliable distances (tf, FP, bs, gc). The typical distance error is about $10 \%$ in the first sub-sample, and about $25 \%$ in the second one. The distribution of galaxies of these two categories by distances is shown in the lower panel of the Fig. 2. About 3/4 of galaxies with accurate distances lie in a virial interval of (14-18) Mpc, while this rate is only about $1 / 4$ among galaxies with distances

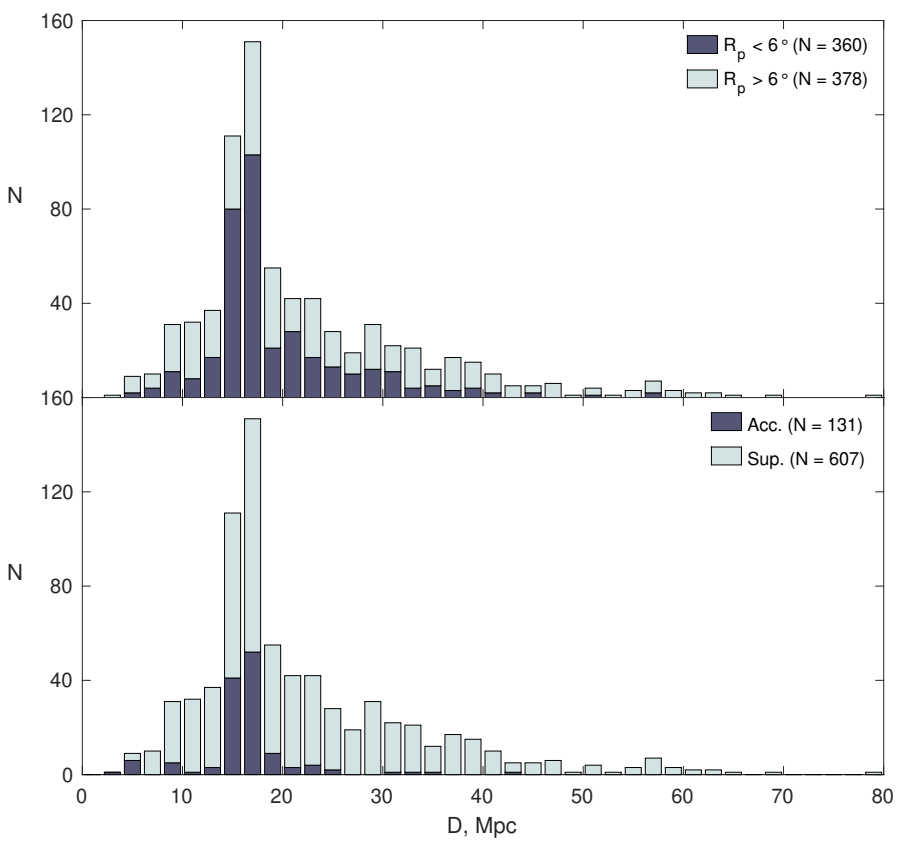

Fig. 2. Distribution of galaxies in Virgo cluster area by their distances. Upper panel: the galaxies of the virial core are marked with a darker colour. Lower panel: the galaxies with "accurate" distances (sbf, cep, $\mathrm{SN}, \mathrm{rgb}$ ) are shown with a darker colour, while those with less reliable ("supplementary") distances (tf, FP, bs, gc) are shown with a lighter colour.

estimated from secondary indicators. It should be noted that this difference is caused not only by the order of distance errors, but also by the morphological segregation: the surfacebrightness-fluctuation method is applied to E, S0, and $\mathrm{dSph}$ galaxies populating the centre of the cluster, while Tully-Fisher relation is suitable for gas-rich S, I, and BCD galaxies inhabiting some less dense regions.

The distribution of galaxies within different distance intervals over the sky is presented in Fig. 3. We enlarged the interval containing Virgo members up to $(12-22) \mathrm{Mpc}$ to account not only for virial depth of the cluster $( \pm 2 \mathrm{Mpc})$, but also for distance errors inherent in the Tully-Fisher method $( \pm 4 \mathrm{Mpc})$. The surface density distribution is smoothed with a window of $0.75^{\circ}$. The circle corresponds to the virial radius of $6^{\circ}$. As can be seen from these data, the foreground galaxies in the upperleft panel do not demonstrate any clumping towards the cluster centre. Most galaxies with distances falling into $(17 \pm 5) \mathrm{Mpc}$ are located in the sky within the virial radius. The background galaxies with $D>22 \mathrm{Mpc}$ group along the filament, which includes several clumps (Kim et al. 2016), the most rich of them are $W$ and $M$ clouds according to de Vaucouleurs. The main galaxies in these clouds (Makarov \& Karachentsev 2011), their coordinates, the mean radial velocities and distances are listed in Table 2.

At present, half of the galaxies in the considered area with $V_{\mathrm{LG}}<2600 \mathrm{~km} \mathrm{~s}^{-1}$ have not distance estimates. These are predominantly intermediate- and low-luminosity galaxies. As we can see from the bottom-right panel of Fig. 3, about $60 \%$ of these galaxies are inside the virial radius of the Virgo cluster, and the majority of the rest belong to the background $W$ cloud.

\section{Luminosity of the Virgo cluster}

The determination of the total luminosity of the Virgo cluster in $B$ or $K$ band is a non-trivial task, because it requires an accurate 

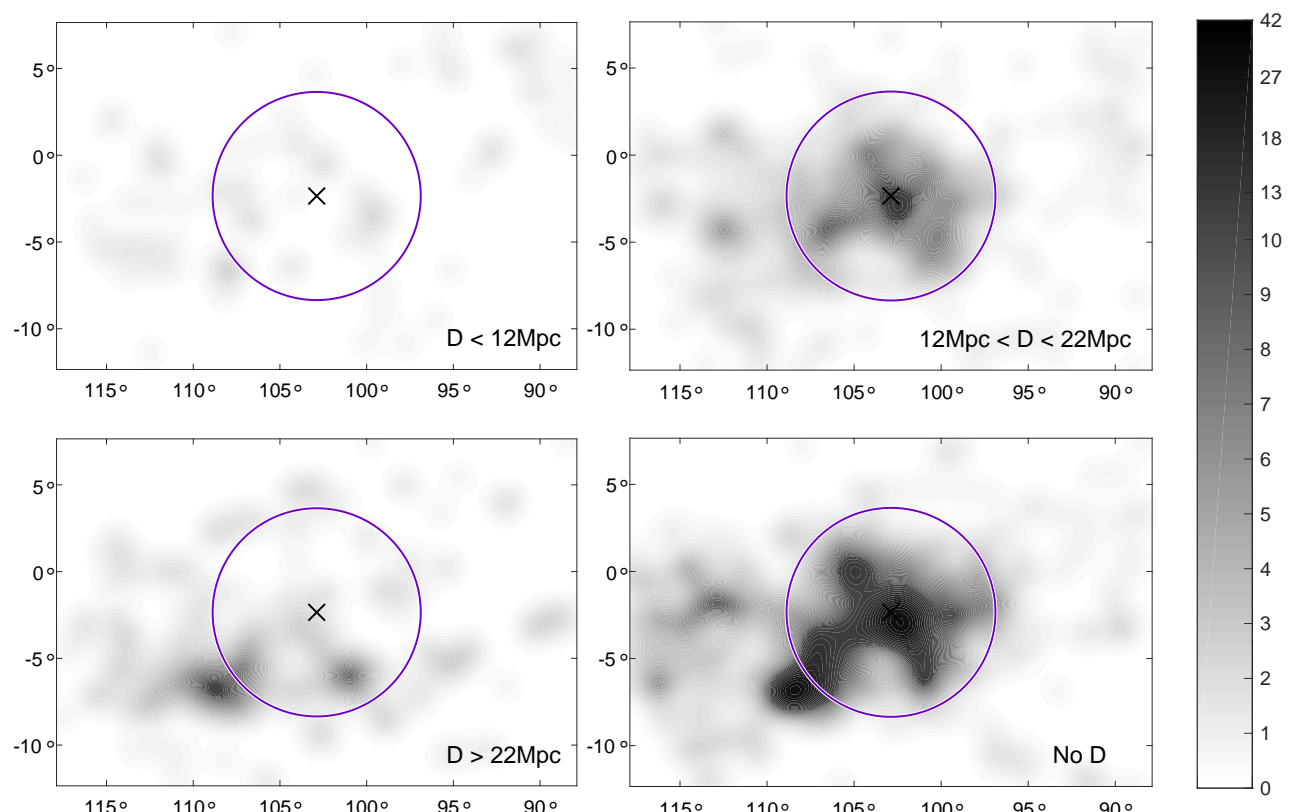

Fig. 3. Sky distribution of galaxies in Virgo cluster area within different distance intervals. The smoothing window is $0.75^{\circ}$. The circle corresponds to the virial radius of $6^{\circ}$.

Table 2. Galaxy clouds behind the Virgo cluster.

\begin{tabular}{lcccccc}
\hline \hline Cloud & Galaxy & $\begin{array}{c}\text { SGL } \\
\left({ }^{\circ}\right)\end{array}$ & $\begin{array}{c}\text { SGB } \\
\left({ }^{\circ}\right)\end{array}$ & $N_{\mathrm{V}}$ & $\begin{array}{c}\left\langle V_{\mathrm{LG}}\right\rangle \\
\left(\mathrm{km} \mathrm{s}^{-1}\right)\end{array}$ & $\begin{array}{c}\langle D\rangle \\
(\mathrm{Mpc})\end{array}$ \\
\hline$W$ & NGC 4261 & 108.4 & -6.9 & 87 & 2060 & 29.4 \\
$M$ & NGC 4189 & 100.7 & -6.0 & 6 & 1987 & 30.0 \\
\hline
\end{tabular}

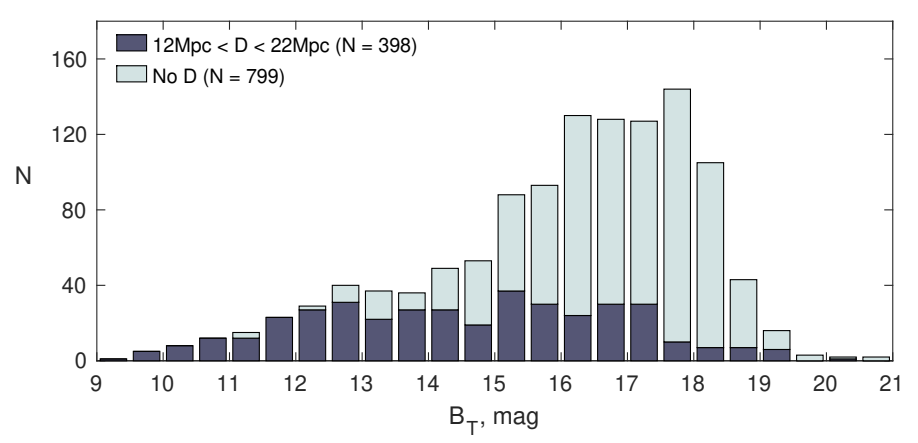

Fig. 4. Distribution of galaxies in Virgo cluster area with measured radial velocities by the apparent magnitude $B_{\mathrm{T}}$. The galaxies with distances $D=(12-22) \mathrm{Mpc}$ are shown with a darker colour, while those with no distances are shown with a lighter colour.

knowledge of cluster membership. The SDSS spectral survey Abazajian et al. (2009) and the ALFALFA survey in $21 \mathrm{~cm}$ HI line (Haynes et al. 2018) have significantly enriched the sample of galaxies with measured radial velocities in the Virgo region. The reasonable completeness of surveys in radial velocities has already been achieved for $B \simeq 16^{\mathrm{m}}-17^{\mathrm{m}}$ corresponding to luminosity of a dwarf galaxy $\left(M_{B} \simeq-15^{\mathrm{m}}-14^{\mathrm{m}}\right)$ at the Virgo distance. However, the radial velocity can't be a reliable indicator of distance in the vicinity of a massive attractor. Morphological type and angular distance from the cluster centre are usually regarded as additional markers, confirming that galaxies are members of a cluster.

Figure 4 represents the distribution of galaxies with distances $D=(12-22) \mathrm{Mpc}$ by $B_{\mathrm{T}}$. The lighter colour indicates galaxies from Table 1 with distances that have not yet been measured.
As one can see, about $90 \%$ of the galaxies with $B_{\mathrm{T}}<13$ mag have distance estimates. These bright galaxies with $M_{B}<-18^{\mathrm{m}}$ make a prevailing contribution to the integrated luminosity of the cluster. We estimated the total luminosity of the galaxies with $D=(12-22) \mathrm{Mpc}$ located inside the $6^{\circ}$ radius to be $L_{B}=$ $(1.8 \pm 0.2) \times 10^{12} L_{\odot}$. Assuming the Schechter luminosity function with the slope parameter $\alpha=-1.3$ (Sandage et al. 1985), we estimated that about $25 \%$ of this quantity is contributed by faint galaxies with as yet unmeasured distances. Therefore, we fix $1.8 \times 10^{12} L_{\odot}$ as an estimate of blue luminosity of the Virgo cluster core. The similar calculations applied to the distribution of galaxies by their $K$-magnitudes yield the total luminosity of the cluster core as $L_{K}=(8.6 \pm 1.1) \times 10^{12} L_{\odot}$. These quantities can be compared with the data from literature. Thus, Sandage et al. (1985) derived the total blue luminosity of the Virgo galaxies within $6^{\circ}$ core to be $1.4 \times 10^{12} L_{\odot}$ at the distance of 16.5 Mpc. According to Kourkchi \& Tully (2017), the galaxies in the whole Virgo association have an integrated $K$-band luminosity of $1 \times 10^{13} L_{\odot}$, with $5 \times 10^{12} L_{\odot}$ in the collapsed core.

\section{Morphological structure}

The relation between morphology and kinematics of galaxies in the Virgo cluster has been studied by de Vaucouleurs \& de Vaucouleurs (1973), Tully \& Shaya (1984), Binggeli et al. (1987, 1993), Bothun \& Mould (1988), Gallagher \& Hunter (1989), Ferguson (1992), Conselice et al. (2001), Ferrarese et al. (2012), and many other authors. In general, early-type gasdeficient galaxies (E, S0, dSp) concentrate at the cluster centre to a much greater degree than spiral and irregular gas-rich galaxies. Schindler et al. (1999) analysed the distribution of the $\mathrm{X}$-ray emission in the Virgo cluster and estimated the mass of the hot intracluster gas to be three times the integral mass of the galaxies. The Sunyaev-Zeldovich effect measurements (Planck Collaboration Int. XL 2016) confirmed the presence of the hot intracluster gas with a total mass of about $1.5 \times 10^{14} M_{\odot}$ being an order of magnitude higher than the integral stellar mass. Peripheral galaxies lose their gas interacting with the intracluster gas medium while falling towards the cluster centre. The loss of gas causes the suppression of the star formation process in galaxies along with their morphological transformation. 

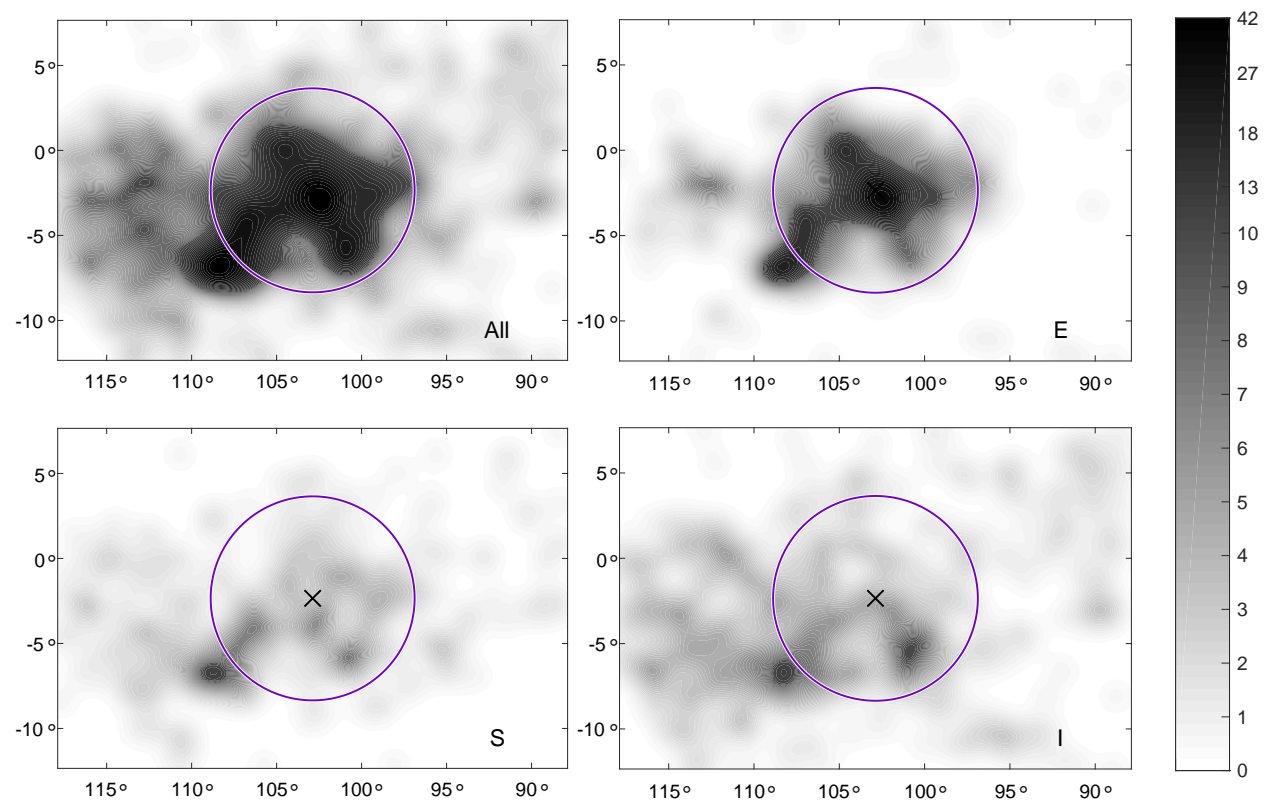

Fig. 5. Sky distribution of galaxies of different morphological types with $V_{\mathrm{LG}}<$ $2600 \mathrm{~km} \mathrm{~s}^{-1}$ in the Virgo cluster area. The smoothing window is $0.75^{\circ}$. The circle corresponds to the virial radius of $6^{\circ}$.

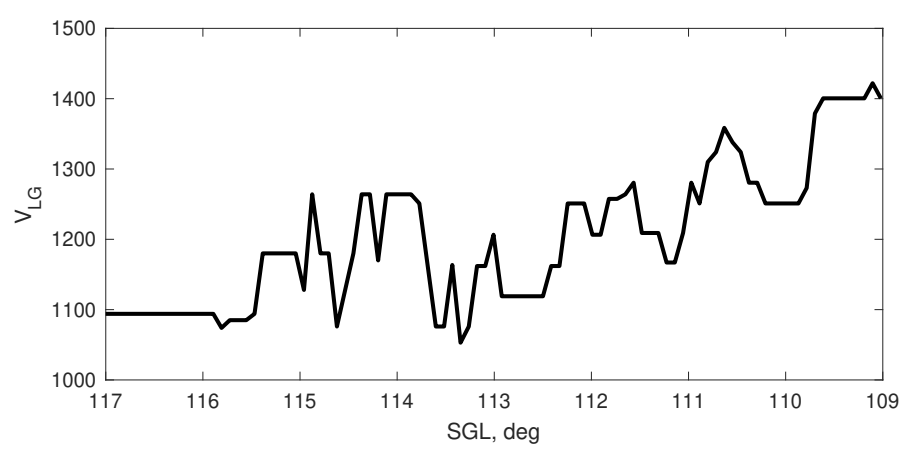

Fig. 6. Radial velocities of galaxies in Virgo Southern Extension as a function of supergalactic longitude. The broken line represents the running median with a window of $2^{\circ}$.

The four panels of Fig. 5 reproduce the distribution of all the galaxies with $V_{\mathrm{LG}}<2600 \mathrm{~km} \mathrm{~s}^{-1}$, as well as early-type galaxies (E, S0, dSph), spiral galaxies, and irregular dwarfs (I, Im, BCD) among them. Their distribution is smoothed with a window of $0.75^{\circ}$. As is clear from these data, the major part of early type galaxies reside within the virial radius $R_{\mathrm{p}}=6.0^{\circ}$. Half of the spiral galaxies lie outside the virial zone, and many of them are associated with background $W$ cloud. The distribution of $S$ galaxies is clumpy inside the virial radius, pointing out that this sub-system has not yet been dynamically relaxed, as noticed by Conselice et al. (2001). Many late-type dwarf galaxies are concentrated in the background $W$ and $M$ clouds, while a significant number of them reside in the Virgo Southern Extension region left of the virial zone. Tully \& Shaya (1984) considered this region as the main channel providing the virial core of the Virgo cluster with extrinsic galaxies.

The evidence of this assumption can be seen in Fig. 6, illustrating the radial velocities of galaxies in the VirgoSE zone and the behaviour of the running median with a window of $2^{\circ}$. The characteristic radial velocity of galaxies grows from $1100 \mathrm{~km} \mathrm{~s}^{-1}$ at the left (southern) border of the considered region $\left(\mathrm{SGL}=117^{\circ}\right)$ up to $1400 \mathrm{~km} \mathrm{~s}^{-1}$ at the virial boundary of the cluster $\left(\mathrm{SGL}=109^{\circ}\right)$. The majority of these galaxies are located between the observer and the Virgo cluster (see the upperleft panel of Fig. 3). While falling along the VirgoSE filament
Table 3. Mean radial velocity $\left\langle V_{\mathrm{LG}}\right\rangle$ and radial velocity dispersion $\sigma_{\mathrm{V}}$ for the different galaxy populations within a (12-22) Mpc distance range.

\begin{tabular}{lccc}
\hline \hline Type & $N$ & $\begin{array}{c}\left\langle V_{\mathrm{LG}}\right\rangle \\
\left(\mathrm{km} \mathrm{s}^{-1}\right)\end{array}$ & $\begin{array}{c}\sigma_{\mathrm{V}} \\
\left(\mathrm{km} \mathrm{s}^{-1}\right)\end{array}$ \\
\hline $\mathrm{E}$ & 103 & 1039 & 516 \\
$\mathrm{~S}$ & 137 & 993 & 674 \\
$\mathrm{I}$ & 158 & 1158 & 676 \\
\hline All & 398 & 1070 & 638 \\
\hline
\end{tabular}

towards the cluster centre, the galaxies with Virgocentric distances within a range of $(2-4) \mathrm{Mpc}$ get an additional line-ofsight velocity component of $\sim 300 \mathrm{~km} \mathrm{~s}^{-1}$ that agrees with the results of $N$-body simulations (Klypin et al. 2003). As far as we know, this is the first direct observational evidence of the case that baryonic matter moves along a cosmic filament toward a cluster as a node of the large-scale structure.

The mean radial velocity $\left\langle V_{\mathrm{LG}}\right\rangle$ and the radial velocity dispersion $\sigma_{\mathrm{V}}$ of $\mathrm{E}, \mathrm{S}$, and $\mathrm{I}$ galaxies with distances in a (12-22) Mpc range are given in Table 3.

The distribution of galaxies of different morphological types by their distance $D$ is shown in three panels of Fig. 7. In these histograms, galaxies located inside the virial radius of $6^{\circ}$ are marked with the darker colour. We can make several conclusions analysing the presented data: (a) Early-type galaxies show a sharp peak within $D=(14-18)$ Mpc distance range, with a tail at $D=(20-30)$ Mpc region caused by $W$ and $M$ clouds members. (b) Spiral galaxies inside $R_{\mathrm{p}}=6.0^{\circ}$ hit a peak within (14-18) Mpc too, but the most spirals lie substantially further away than the Virgo cluster. Among the spirals residing within the $6^{\circ}$ radius, only half have distances that are typical for Virgo. (c) Of late-type dwarf galaxies, only $1 / 3$ are associated with the virial core of the cluster; the main population of gas-rich dwarfs lies in the background or foreground relative to the cluster.

\section{Density profiles and total mass of the cluster}

The distribution of surface density (per square degree) of Virgo galaxies along the projected radius $R_{\mathrm{p}}$ of the cluster is presented 


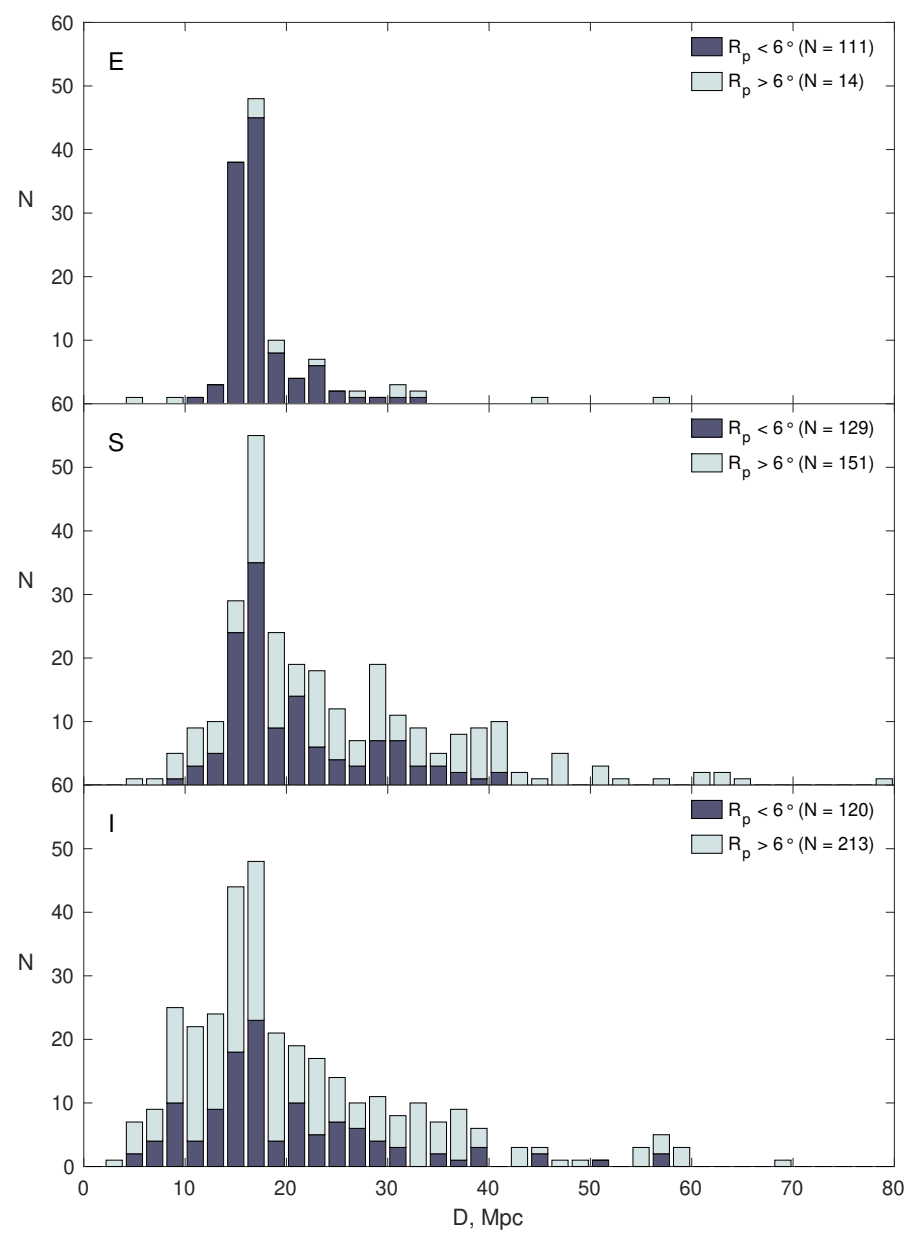

Fig. 7. Distribution of galaxies of different morphological types in the Virgo cluster area by their distance $D$. The galaxies within the $6^{\circ}$ core are marked by a darker colour.

in the logarithmic scale in Fig. 8. To plot this density profile, we used only the galaxies with distances in a (12-22) Mpc range. The solid line corresponds to the standard projected NavarroFrenk-White (NFW) profile of the dark halo (Navarro et al. 1997; Bartelmann 1996) with a fitted scale radius $R_{\mathrm{s}}=4.3^{\circ}$ or $1.24 \mathrm{Mpc}$ in projection. One can notice that there is an abrupt edge at $R_{\text {edge }}=6.5^{\circ}$ that is inferred to correspond to the projected virial radius. Using the data listed in Table 1 , we determined the mean, $\left\langle R_{\mathrm{p}}\right\rangle$, and the median for projected distances of galaxies from M 87. The data are presented in Table 4 for wide and narrow ranges of distances: (12-22) Mpc and (14-20) Mpc. We can see that the $\left\langle R_{\mathrm{p}}\right\rangle$ uncertainty caused by various choices of cluster members is not large and does not differ significantly with statistical error $(\sim 5 \%)$.

According to Tully (2015), the virial mass of a cluster $M_{\mathrm{vir}}$ is expressed via the radial velocity dispersion $\sigma_{\mathrm{p}}$ and the projected radius $R_{g}$ as

$M_{\mathrm{vir}}=(\alpha \times \pi / 2) G^{-1} \times \sigma_{\mathrm{p}}^{2} \times R_{g}$,

where $G$ is gravitational constant, projected radius $R_{g}$ is the mean harmonic distance for all the pairs, and the dimensionless parameter $\alpha \simeq 2.5 \pm 0.1$ accounts for the conversion of radial velocity dispersion into $3 \mathrm{D}$ velocity dispersion under assumption of weak anisotropy. It should be noted, however, that the mean harmonic distance between galaxies is not a robust characteristic of a cluster radius due to projection effects. Assuming

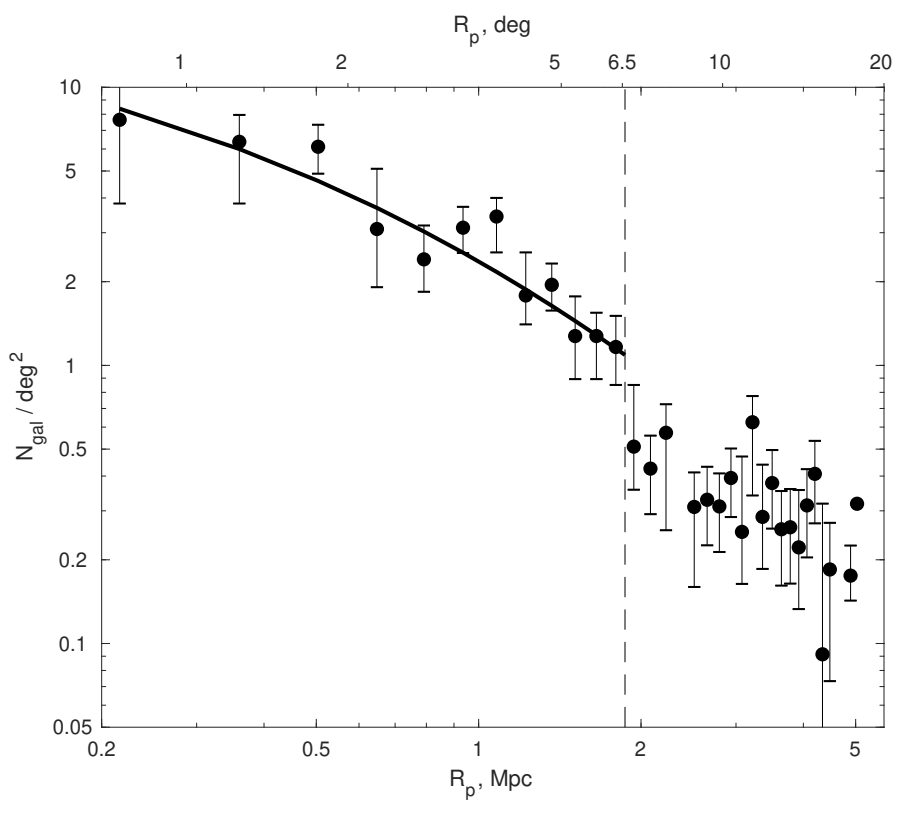

Fig. 8. Surface density profile of Virgo galaxies as a function of projected radius $R_{\mathrm{p}}$ given in degrees (upper scale) and Mpc (lower scale). The solid line corresponds to the standard projected NFW profile of the dark halo.

Table 4. Median and average separation of galaxies from M 87.

\begin{tabular}{lccc}
\hline \hline $\begin{array}{l}D \\
(\mathrm{Mpc})\end{array}$ & $N_{\mathrm{D}}$ & Median & $\left\langle R_{\mathrm{p}}\right\rangle$ \\
\hline $12-22$ & 398 & $4.74^{\circ}$ & $5.91 \pm 0.20^{\circ}$ \\
$14-20$ & 316 & $4.36^{\circ}$ & $5.69 \pm 0.23^{\circ}$ \\
\hline
\end{tabular}

the mean distance of the cluster $16.5 \mathrm{Mpc}$, radial velocity dispersion $\sigma_{\mathrm{p}}=638 \mathrm{~km} \mathrm{~s}^{-1}$ (see below) and $R_{g}$ in the range between $\left\langle R_{\mathrm{p}}\right\rangle=5.7^{\circ}$ and $R_{\text {edge }}=6.5^{\circ}$, we get the following virial mass estimation for the Virgo cluster:

$M_{\mathrm{vir}}=(6.0-6.8) \times 10^{14} M_{\odot}$.

An elegant way of determining the mass of a spherically symmetrical cluster was proposed by Ambartsumian (1939). The potential energy of a system $U$ is expressed from the density distribution $F(y)$ projected into a line crossing the cluster centre as

$U=G \int_{-\infty}^{+\infty}[F(y)]^{2} \mathrm{~d} y$

The problem of finding $U$ can be reduced to galaxy number counts in parallel stripes of $\Delta y$ width. Substituting Eq. (3) in the virial theorem $2 T+U=0$ where $T$ is kinetic energy, we obtain the expression for the total mass of the cluster

$M_{\mathrm{vir}}=v \alpha \times G^{-1} \times \sigma_{\mathrm{p}}^{2} \times \Delta y$.

The dimensionless factor

$v=\frac{\left(\Sigma n_{i}\right)^{2}}{\Sigma n_{i}^{2}}$

is governed by the galaxy number counts, $n_{i}$, in parallel stripes of $\Delta y$ width, and another dimensionless factor $\alpha \simeq 2.5$, like in Eq. (1), accounts for conversion of radial velocity dispersion into the full velocity dispersion. 

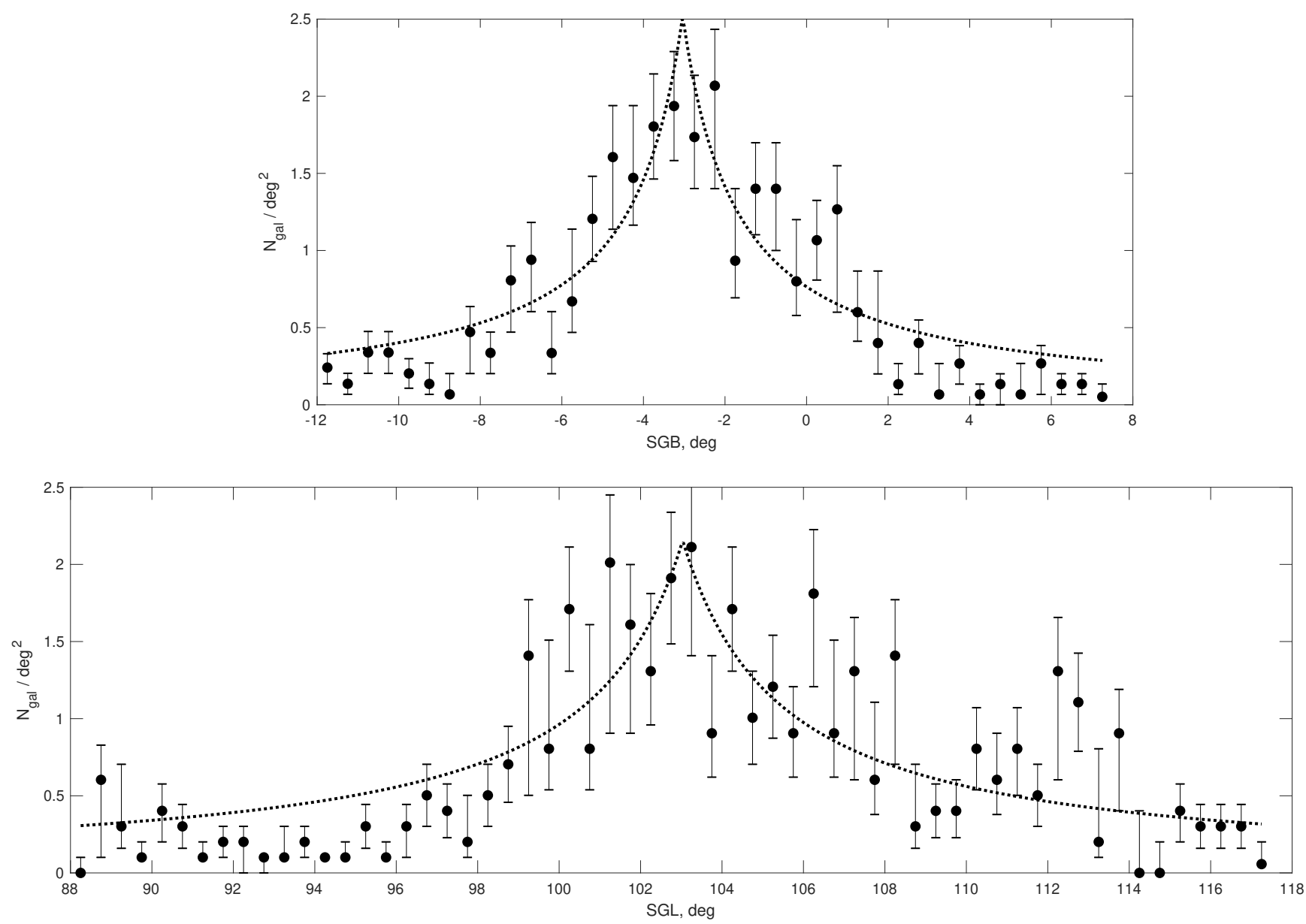

Fig. 9. Standard one-dimensional NFW profile for Virgo galaxy counts with estimated parameters and 95\% confidence intervals.

Table 5. Galaxy counts in strips.

\begin{tabular}{lccc}
\hline \hline Strips & & $v$ & $\begin{array}{c}M \\
\left(10^{14} \times M_{\odot}\right)\end{array}$ \\
\hline Along SGL, & $\Delta y=0.5^{\circ}$ & 19.5 & 6.6 \\
& $\Delta y=1.0^{\circ}$ & 10.9 & 7.4 \\
Along SGB, & $\Delta y=0.5^{\circ}$ & 16.1 & 5.4 \\
& $\Delta y=1.0^{\circ}$ & 8.4 & 5.7 \\
\hline
\end{tabular}

The first galaxy counts in stripes, arranged parallel and perpendicular to the declination, yielded $6.9 \times 10^{14} M_{\odot}$ and $9.3 \times 10^{14} M_{\odot}$, respectively, for the mass of the Virgo cluster (Karachentsev 1965). We repeated galaxy counts in the Virgo cluster within a (12-22) Mpc distance range. The counts were performed in stripes of $\Delta y=0.5^{\circ}$ and $1^{\circ}$ width, oriented along SGB and SGL. The results are shown in the two panels of Fig. 9. The obtained values of $v$ factor and the total mass of the cluster are presented in Table 5. The difference in counts made along SGL and SGB are quite predictable since the Virgo cluster is non-spherical and elongated by the LSC equator. The mean value of mass from four estimates is

$M=(6.3 \pm 0.9) \times 10^{14} M_{\odot}$,

in good agreement with the mass estimation obtained in Eq. (2) from the projected radius. The uncertainty of the mass includes the uncertainty of radial velocity dispersion.
Assuming the standard NFW spatial density profile (Navarro et al. 1997) for the dark halo of the cluster as

$D(x)=D_{\mathrm{c}} / x \times(1+x)^{2}$,

where $D_{\mathrm{c}}$ is a characteristic density and $x=r / r_{\mathrm{s}}$ is a dimensionless distance expressed in units of a scale radius $r_{\mathrm{s}}$, we obtain a simple equation for density function projected onto the axis:

$F(y)=\frac{F_{0}}{1+y}$,

as, by definition,

$F^{\prime}(x)=-2 \pi x \times D(x)$.

This standard 1D NFW profile is represented in two panels of Fig. 9 with dotted hyperbola. As we can see, the standard density profile looks much sharper than the real profile of galaxy counts along SGB and SGL. The Kolmogorov-Smirnov test for goodness-of-fit gives the evidence of non-normally distributed residuals with $p$-values of $3.8 \times 10^{-5}$ and $1.4 \times 10^{-4}$ for galaxy counts along SGL and SGB, respectively, suggesting a very weak agreement between the observed distribution of galaxies and the expected density distribution. In contrast to this, McLaughlin (1999) found that the "universal" NFW model by Navarro et al. (1997) traces the galaxy distribution in the Virgo cluster very well, yielding its virial mass of $(4.2 \pm 0.5) \times 10^{14} M_{\odot}$.

We note that Simionescu et al. (2017) investigated X-ray properties of the Virgo cluster with SUZAKU Key Project and 


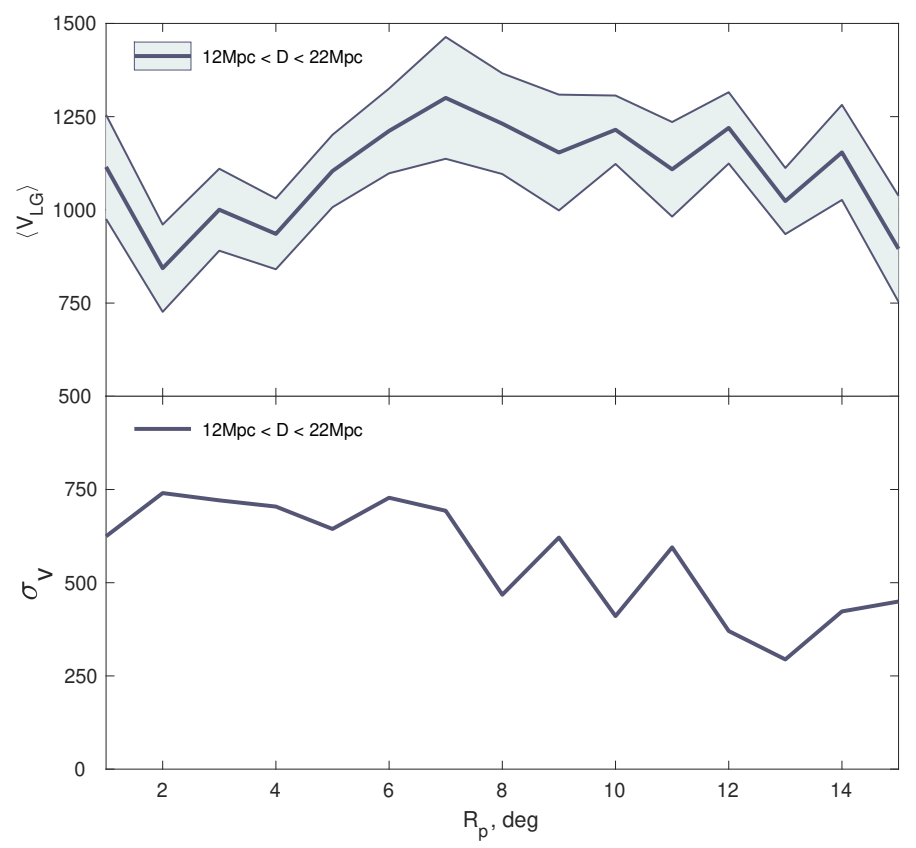

Fig. 10. Mean radial velocity $\left\langle V_{\mathrm{LG}}\right\rangle$ and radial velocity dispersion $\sigma_{\mathrm{V}}$ of Virgo galaxies as a function of projected radius.

obtained a much lover virial radius, $r_{200}=3.36^{\circ}$, and virial mass estimate, $M_{200}=1.05 \times 10^{14} M_{\odot}$, assuming isothermal hot gas to be in hydrostatic equilibrium within a NFW potential (Navarro et al. 1997).

Finally, it should be stressed that we assume the Virgo cluster is virialised. However, as Conselice et al. (2001) demonstrated, only elliptical galaxies in the Virgo core region form a nearly relaxed subsystem, no other galaxy populations (spiral or irregular) have characteristics of relaxation. Also, we assume that the galaxy orbit distribution has a weak anisotropy. There is, however, ample evidence that many spiral and irregular galaxies move across the cluster core in elongated, almost radial orbits. This makes the estimation of the total velocity dispersion of galaxies dependent on the morphological composition of the sample. Obviously, these simplifying assumptions affect the estimation of the virial mass and its errors.

If we suppose that only early-type galaxies with their radial velocity dispersion of $516 \mathrm{~km} \mathrm{~s}^{-1}$ are in the relaxed state, the virial mass of the Virgo cluster turns out to be $4.1 \times 10^{14} M_{\odot}$, in close agreement with the McLaughlin (1999) estimate. Also, replacing the virial theorem $2 T+U=0$ in the condition of negative total energy of the cluster, $T+U \leq 0$, yields us the estimate of the total cluster mass $M_{\mathrm{T}} \geq M_{\mathrm{vir}} / 2$.

As mentioned above, the Planck Collaboration Int. XL (2016) performed a detailed study of the Virgo cluster through Sunyaev-Zeldovich effect and found the total mass of warm/hot gas to be $(1.4-1.6) \times 10^{14} M_{\odot}$. Assuming the cosmic value for the baryon fraction, $f_{\mathrm{b}}=\Omega_{\mathrm{b}} / \Omega_{\mathrm{m}}=0.1834$, they found that the total mass of the cluster would be $(7.6-8.7) \times 10^{14} M_{\odot}$ on a scale up to 2 times larger than the virial radius.

\section{Basic parameters of the Virgo cluster}

The nearest cluster of galaxies in Virgo is qualified as a rather rich one, though it is not included in the known catalogues by Zwicky et al. (1961) and Abell (1958) due to its large angular size. The existing substructures around M 87, M 49, M 86, and other bright galaxies, were extensively distinguished (de Vaucouleurs 1961;
Table 6. Average parameters of the Virgo cluster.

\begin{tabular}{lcl}
\hline \hline Parameter & $\begin{array}{c}\text { Derived } \\
\text { value }\end{array}$ & Unit \\
\hline$\left\langle V_{\mathrm{LG}}\right\rangle$ & $1070 \pm 48$ & $\mathrm{~km} \mathrm{~s}^{-1}$ \\
$\sigma_{\mathrm{p}}$ & $638 \pm 35$ & $\mathrm{~km} \mathrm{~s}^{-1}$ \\
$\langle D\rangle$ & $16.5 \pm 0.2$ & $\mathrm{Mpc}$ \\
$R_{g}$ & $1.7 \pm 0.2$ & $\mathrm{Mpc}$ \\
$L_{B}$ & $1.8 \pm 0.2$ & $10^{12} L_{\odot}$ \\
$L_{K}$ & $8.6 \pm 1.1$ & $10^{12} L_{\odot}$ \\
$M_{\mathrm{vir}}$ & $6.3 \pm 0.9$ & $10^{14} M_{\odot}$ \\
\hline
\end{tabular}

Schindler et al. 1999; Mei et al. 2007; Kim et al. 2014) as being illustrative of incomplete dynamical relaxation of the Virgo cluster. The observable infall of late-type galaxies to the virial zone along the VirgoSE filament also indicates this fact. The Virgo cluster, being the most broadly studied one, is involved in a comparison of the numerical simulation results with the observations. This is the reason why it would be instructive to compile an "identity card" of the cluster containing its principal observational characteristics.

Two panels of Fig. 10 illustrate the dependence of the cluster mean radial velocity and the radial velocity dispersion on the projected distance from M 87 with a step of $1^{\circ}$. The analysis introduces galaxies in a (12-22) Mpc distance range. The variations of the mean radial velocity seem to be significant and appreciably exceed the SEM error bars. Besides the virial core, $R_{\mathrm{p}}>6^{\circ}$, the observable variations are caused mainly by the kinetic pattern of the ongoing infall of galaxies towards the cluster centre. The decreasing trend of radial velocity dispersion towards the outskirts of the cluster bears evidence of mainly radial flows of galaxies. The mean value of radial velocity is $\left\langle V_{\mathrm{LG}}\right\rangle=1070 \pm 48 \mathrm{~km} \mathrm{~s}^{-1}$ for the virial zone of the cluster, and the corresponding radial velocity dispersion is $638 \pm 35 \mathrm{~km} \mathrm{~s}^{-1}$. Assuming $16.5 \pm 0.2 \mathrm{Mpc}$ as the cluster distance and $H_{0}=$ $73 \mathrm{~km} \mathrm{~s}^{-1} \mathrm{Mpc}^{-1}$ as the local Hubble parameter, we obtain the value $-135 \pm 50 \mathrm{~km} \mathrm{~s}^{-1}$ as the Virgocentric infall velocity of the Local Group.

The principal integral characteristics of the Virgo cluster are listed in Table 6. In most cases, the cited errors of mean values are formal, since there are different underlying factors that affect the estimate of the mean but are difficult to quantify.

\section{Concluding remarks}

The analysis of galaxy motions in the outskirts of the Virgo cluster makes it possible to measure the radius of the zero-velocity surface, $R_{0}=7.0-7.3 \mathrm{Mpc}$ (Karachentsev et al. 2014; Shaya et al. 2017; Kashibadze et al. 2018), corresponding to the total mass of the Virgo cluster $M_{\mathrm{T}}=(7.4 \pm 0.9) \times 10^{14} M_{\odot}$ inside the $R_{0}$. The numerical simulated trajectories of nearby galaxies with accurate distance estimates performed by Shaya et al. (2017) confirmed the obtained estimate of the total mass of the cluster. The virial mass of the cluster, being determined independently at the scale of $R_{g}=1.7 \mathrm{Mpc}$ from the internal motions, is nearly the same $-M_{\text {vir }}=(6.3 \pm 0.9) \times 10^{14} M_{\odot}$. The agreement of internal and external mass estimates within their nominal error of $\sim 15 \%$ attests to the fact that the wide outskirts of the Virgo cluster between $R_{g}$ and $R_{0}$ radii do not contain significant amounts of dark matter. This observational result seems to be a challenge for certain cosmological models within the standard $\Lambda$ CDM paradigm, affirming that the peaks of dark matter 
large-scale distribution are less sharp than the stellar matter peaks (Fukugita \& Boehringer 2019). The explanation of this controversy ${ }^{4}$ requires new observational ${ }^{5}$ and theoretical efforts.

Acknowledgements. We thank the anonymous referee whose suggestions and comments helped to improve significantly the presentation of the paper results. The work is supported by the RFBR grant No. 18-02-00005. We acknowledge the usage of SDSS, PanSTARRS and ALFALFA surveys, as well as the HyperLeda and NED databases.

\section{References}

Abazajian, K. N., Adelman-McCarthy, J. K., Agueros, M. A., et al. 2009, ApJS, 182,543

Abell, G. 1958, ApJS, 3, 211

Ambartsumian, V. A. 1939, Doklady Akademii Nauk SSSR, 24, 875

Bartelmann, M. 1996, A\&A, 313, 697

Binggeli, B., Sandage, A., \& Tammann, G. A. 1985, AJ, 90, 1681

Binggeli, B., Tammann, G. A., \& Sandage, A. 1987, AJ, 94, 251

Binggeli, B., Popescu, C. C., \& Tammann, G. A. 1993, A\&AS, 98, 275

Bothun, G. D., \& Mould, J. R. 1988, ApJ, 324, 123

Cantiello, M., Blakeslee, J. B., Ferrarese, L., et al. 2018, ApJ, 856, 126

Chambers, K. C., Magnier, E. A., Metcalfe, N., et al. 2016, ArXiv e-prints [arXiv:1612.05560]

Conselice, C. J., Gallagher, III, J. S., \& Wyse, R. F. G. 2001, ApJ, 559, 791

de Vaucouleurs, G. 1961, ApJS, 6, 213

de Vaucouleurs, G., \& de Vaucouleurs, A. 1973, A\&A, 28, 109

Ferguson, H. C. 1992, MNRAS, 255, 389

Ferrarese, L., Cote, P., Cuillandre, J. C., et al. 2012, ApJS, 200, 4

Fukugita, M., \& Boehringer, H. 2019, MNRAS, 490, 1693

Gallagher, III, J. S., \& Hunter, D. A. 1989, AJ, 98, 806
Haynes, M. P., Giovanelli, R., Martin, A. M., et al. 2011, AJ, 142, 170 Haynes, M. P., Giovanelli, R., Kent, B. R., et al. 2018, ApJ, 861, 49 Jarrett, T. H., Chester, T., Cutri, R., et al. 2003, AJ, 125, 525 Karachentsev, I. D. 1965, Astrophysics, 1, 303

Karachentsev, I. D., \& Kashibadze, O. G. 2010, Astrophysics, 53, 32

Karachentsev, I. D., Tully, R. B., Wu, P. F., et al. 2014, ApJ, 782, 4

Karachentsev, I. D., Kaisina, E. I., \& Kashibadze, O. G. 2017, AJ, 153, 6

Kashibadze, O. G., Karachentsev, I. D., \& Karachentseva, V. E. 2018, Astrophys. Bull., 73, 124

Kim, S., Rey, S. C., Jerjen, H., et al. 2014, ApJS, 215, 22

Kim, S., Rey, S. C., Bureau, M., et al. 2016, ApJ, 833, 207

Klypin, A., Hoffman, Y., Kravtsov, A. V., \& Gottloeber, S. 2003, ApJ, 596, 19

Kourkchi, E., \& Tully, R. B. 2017, ApJ, 843, 16

Makarov, D. I., \& Karachentsev, I. D. 2011, MNRAS, 412, 2498

Makarov, D., Prugniel, P., Terekhova, N., et al. 2014, A\&A, 570, A13

McLaughlin, D. E. 1999, ApJ, 512, L9

Mei, S., Blakeslee, J. P., Côte, P., Tonry, J. L., et al. 2007, ApJ, 655, 144

Navarro, J. F., Frenk, C. S., \& White, S. D. M. 1997, ApJ, 490, 493

Planck Collaboration Int. XL. 2016, A\&A, 596, A101

Sandage, A. R., \& Tammann, G. A. 1981, A Revised Shapley-Ames Catalog of Bright Galaxies (Washington: Carnegie Institution)

Sandage, A. R., Binggeli, B., \& Tammann, G. A. 1985, AJ, 90, 1759

Schindler, S., Binggeli, B., \& Boehringer, H. 1999, A\&A, 343, 420

Shaya, E. J., Tully, R. B., Hoffman, Y., \& Pomarede, D. 2017, ApJ, 850, 207

Simionescu, A., Werner, N., Manz, A., et al. 2017, MNRAS, 469, 1476

Toloba, E., Lim, S., Peng, E., et al. 2018, ApJ, 856, L31

Tonry, J. L., Blakeslee, J. P., Ajhar, E. A., \& Dressler, A. 2000, ApJ, 530, 625

Tully, R. B. 2015, AJ, 149, 171

Tully, R. B., \& Fisher, J. R. 1977, A\&A, 54, 661

Tully, R. B., \& Pierce, M. J. 2000, ApJ, 533, 744

Tully, R. B., \& Shaya, E. J. 1984, ApJ, 281, 31

Zwicky, F., Herzog, E., \& Wild, P. 1961, Catalogue of Galaxies and of Clusters of Galaxies (Pasadena: California Institute of Technology), 1

\footnotetext{
4 http://leda.univ-lyon 1 .fr

5 http://ned.ipac.caltech.edu
} 